\title{
Evaluating Innovation Diffusion Readiness among Architectural and Engineering Design Firms: Empirical Evidence from Australia
}

Kriengsak Panuwatwanich

Griffith School of Engineering, Griffith University

Gold Coast Campus, Queensland 4222, Australia

Contact email: k.panuwatwanich@griffith.edu.au

\section{Rodney A. Stewart}

Griffith School of Engineering, Griffith University Gold Coast Campus, Queensland 4222, Australia

Contact email: r.stewart@griffith.edu.au 


\title{
Evaluating Innovation Diffusion Readiness among Architectural and Engineering Design Firms: Empirical Evidence from Australia
}

\begin{abstract}
This paper conceptualises the development of an Innovation Diffusion Readiness (IDR) framework used for monitoring innovation diffusion maturity levels within the Architectural and Engineering Design (AED) firm context. To achieve this, a survey was conducted with a number of Australian AED firms, using a validated survey instrument developed elsewhere. The key findings based on the study sample of 158 professionals representing 44 AED firms showed that the overall level of IDR among the sampled firms was moderate to high. Using Cluster Analysis, three main clusters of these firms that shared common IDR patterns were uncovered: Very High, High and Moderate IDR. Further analysis indicated that clusters with higher IDR appeared to perform significantly better in diffusing innovative design practices (i.e. value-based innovations). This finding highlighted that firms shifting to a higher IDR cluster could achieve a significant improvement in the diffusion of this type of innovation. The paper also provided a number of recommendations for future research to further improve the assessment of IDR within AED firms.
\end{abstract}

\section{Keywords}

Australia; architect; cluster analysis; design; innovation diffusion; readiness; engineer 


\section{Introduction}

Innovation plays a key role in leveraging the competitiveness of firms within the construction industry, in particular, Architectural and Engineering Design (AED) firms. According to Torbett [1], design is essentially a combination of creativity, intellectual content, technical possibilities and market demand. Design is also a complex social activity, thus conceiving innovative design solutions requires a great deal of collaboration and communication among participating members [2,3]. A case study of a large British engineering organisation conducted by Salter et al. [3] revealed that most of the ideas for design innovations came from a combination of personal interactions between designers and the use of advanced information and communication technology (ICT) tools. This finding can be associated with the notion that design knowledge is often embedded in individuals and organisational processes [1]. Therefore, AED firms that have the ability to encourage creativity among individual designers and harness innovative ideas from them, as well as the ability to adopt appropriate systems and tools to help diffuse such ideas, are more likely to achieve innovative design outcomes. According to Panuwatwanich et al. [4], such abilities can be understood through examining the socio-psychological aspect of innovation diffusion. This approach suggests that successful and effective diffusion of innovation is dependent upon the internal environment (structure, policy, culture, resources, etc.) of the firms, which must be such that it is conducive to innovation diffusion. Firms need to examine such factors to determine if they are ready, as forcing innovations can be counterproductive.

Researchers within the field of Architecture, Engineering and Construction (AEC) have widely highlighted the significance of organisation readiness, which is considered as one of the key factors critical to effective implementation and diffusion of various types of innovations. For example, Kamara et al. [5] emphasise the importance of assessing organisation readiness to pinpoint 'resistors' and 'enablers' that would influence the successful implementation of knowledge management within AEC firms. Similarly, by studying the implementation of a cross-sectoral knowledge management process across a number of UK projects, Siemieniuch and Sinclair [6] concluded that organisations should evaluate their "organisation readiness" prior to considering how best to implement appropriate knowledge management process to ensure that organisations achieve the most added-value out of the implemented process. To improve Concurrent Engineering (CE) implementation, Khalfan et al. [7] address the need to incorporate a readiness assessment of an organisation into the implementation 
planning prior to the actual introduction of CE. Correspondingly, Peansupap and Walker [8] state that a lower than expected business outcome derived from ICT investments may result from the lack of understanding of the organisation's true level of ICT readiness, which can lead to overestimation of ICT benefits as a result of inaccurate information obtained regarding organisation reality. In relation to ICT implementation, the concept of e-readiness was also investigated by Ruikar et al. [9], highlighting the need for companies to assess their ability to successfully adopt, use and benefit from ICT (i.e. ereadiness) such as e-commerce. In view of this, it can be argued that the ability of firms to understand their readiness for innovation diffusion is crucial.

The principal aim of the research presented herein is to evaluate and understand the current levels of Innovation Diffusion Readiness (IDR) that exist among the selected group of Australian AED firms. The remaining sections of this paper are organised as follows. The next section provides a theoretical background on the socio-psychological aspect of innovation diffusion and readiness, which leads to the forming of research questions. Methodology adopted in the research is then explained and analysis results presented. Following this are sections that provide the discussion of results and conclude the research findings. The paper ends with directions for future research.

\section{Theoretical background}

2.1 Innovation diffusion from the socio-psychological perspective

Diffusion of innovation (DOI) is a process in which new ideas, concepts, techniques, etc. are communicated through certain channels over time among the members of a social system [10]. This process involves four key elements: (1) innovations; (2) communication channels; (3) time; and (4) a social system. Innovations can be either creative ideas that lead to the development of new or innovative products, or new technologies or practices adopted to improve either the production process or the product itself. Communication channels are the means by which the information (regarding innovations) is shared and exchanged among members of the social system. Time is used to determine the earliness/lateness and rate (i.e. the speed) of diffusion. The last element, a social system, is defined as "a set of interrelated units that are engaged in joint problem solving to accomplish a common goal” [10]. 
According to Rogers (2003), social structure and system norms also play an important role in the diffusion process. A social structure is the patterned arrangements of the social relationships among the members of a system. This social structure can facilitate or impede the diffusion of innovations in such a way that it influences the flow of communication (regarding the innovation information). More specifically, it has been reported that insufficient innovation communication is a frequently overlooked reason for ineffective innovation implementations [11]. System norms are the established behaviour patterns that define a range of tolerable behaviour and serve as a standard for the members of a social system. In organisations, system norms can be represented by such a factor as organisational culture, through the shared perception of the organisation's members (i.e. organisational climate) [12].

To the wider innovation research community, innovation is regarded as a product of social relationships and complex systems of interaction $[13,14]$. Therefore, innovation and diffusion process essentially involve a great deal of social psychology, which plays an important role in fostering the DOI process within an organisation. Social psychology is fundamentally an explanation of how the cognition, thought, feeling, and behaviour of individuals are influenced by the actual, imagined, or implied presence of others [15]. In particular, a manifestation of social influence, namely 'climate for innovation', has been regarded as a major determinant of people's innovative behaviour and motivation to innovate [16-18]. Theoretically, climate can be defined as "a shared and enduring molar perception of the psychologically important aspects of the work environment” [19, p. 837]. According to Amabile et al. [20], psychological perceptions of innovation (e.g. the implementation of people's ideas) within an organisation are likely to impact the motivation to generate new ideas. Dulaimi et al. [21] further explain that climate for innovation plays a major role in activating or inhibiting innovation by transmitting signals to organisational members concerning the expectations for innovation from the organisation.

Within the AEC context, Panuwatwanich et al. [4] identified three socio-psychological constructs that represent the underlying climate for innovation: (1) leadership for innovation; (2) team climate for innovation; and (3) organisational culture for innovation. Based on this conceptualisation, the authors conducted an empirical study to investigate the relationships between these constructs and their impacts on innovation diffusion outcomes and business performance. It was found that 
organisational culture for innovation functions as a gateway to the diffusion of innovation, by mediating the relationships between leadership and team climate for innovation, and innovation diffusion outcomes. More importantly, it was found that all pathways to innovation diffusion outcomes emerged from the leadership for innovation construct, highlighting its critical role in creating an innovative team climate and organisational culture that fosters and nurtures innovation.

\subsection{Organisation readiness for innovation diffusion}

Organisational readiness has been described in innovation literature as the level of preparedness of a firm for adopting and implementing innovation [22]. Because innovation "can precede and cause social change, or it may be developed in response to the needs created by social change” [23, p. 4], understanding organisational readiness for change is important for firms seeking to implement or generate innovations. Additionally, DOI in particular creates a process of social change by which alteration occurs in the structure and function of a social system [10]. Therefore, to adequately accommodate innovation, firms should be able to effectively handle and manage change. In the emerging organisational emotional intelligence concept, Cherniss [24] points out that change usually causes emotional reactions among firms' leaders and other organisational members; therefore firms must be aware of and be able to manage those emotional reactions, and act to help people cope with them. Such an ability to manage change and emotional reactions can influence organisational effectiveness in a number of areas, including innovation [24]. In addition, because the change caused by innovation implies a degree of risks and uncertainties, self-understanding of organisational readiness can help firms to mitigate those risks associated with the innovation [22]. In this way, firms are more likely to be successful in implementing and diffusing innovations.

Past research studies postulated that the indicators of organisational readiness for innovation and change may include such factors as existing staff skill and knowledge, availability of resource, innovation-supportive values and goals, innovation-system fit and tension for change [25,26]. Within a more specific context of AEC, Ruikar et al. [9] proposed an assessment model to evaluate e-readiness for construction companies (VERDICT) in adopting e-commerce technology. This model established that for an organisation to be e-ready, it must have (1) management that drives the adoption, implementation and usage of the technology; (2) processes that are favourable to the successful 
adoption of the technology; (3) people who have belief, knowledge, skills and abilities in the technology; and (4) technology necessary to support the business functions. Similarly, the BEACON model, developed by Khalfan et al. [7] to assess an organisation readiness for concurrent engineering, consists of four main elements: people, project, process and technology.

Whilst the above studies provided valuable approaches to assess organisation readiness for innovation in general, they largely focused only on the adoption of innovation and did not consider the aspects specifically relevant to the DOI theory in particular. Given this, the current research argues that the measure of IDR should be able to gauge a firm's socio-psychological condition that is conducive for DOI. This is mainly because, as mentioned earlier, the effectiveness of DOI depends largely upon socio-psychological processes (i.e. climate for innovation). This viewpoint is corroborated by Hamilton et al. [27] and Lehman et al. [28] who suggest that organisation readiness for innovation and change can be evaluated through examining leadership and organisational climate. Moreover, by integrating the concepts of innovation diffusion, change management, and learning and sharing knowledge, Peansupap and Walker [29] argue for the inclusion of the important dynamic factors of change management that drive the diffusion of innovation (in this case ICT) within an organisation; these factors include motivation, training and technical support, supervisor support and open discussion. In view of this, the current research proposes that using the climate for innovation model [30] cited in the preceding section can provide a viable framework for assessing a firm's readiness for innovation diffusion. The main reason is that such a model includes the measure for three salient socio-psychological constructs (i.e. leadership, team climate and organisational culture), which were developed from well-established theories on DOI, organisational culture, leadership and work group behaviour. This model also considers the diffusion of both product and process innovations rather than a single type (e.g. ICT) as reviewed above. According to Cooper [31] and Zmud [32], product innovation refers to the introduction of new products or services which shift or expand the domain of an organisation; while process innovation refers to the introduction of new methods or procedures within an existing domain. 


\subsection{Research questions}

Within the AEC context, the emphasis of innovation as a source of competitive advantage has been on successful development and/or implementation of new ideas, products, processes or practices to increase an organisation’s efficiency and performance [33-35]. Invariably, successful development and implementation of innovation requires effective bottom-up and top-down diffusion processes, respectively [36,37]. As a result, effective diffusion processes should be in place to ensure that the implemented innovations help contribute to a firm's desired performance. A number of past research studies in this field have attempted to identify the drivers for innovation (e.g. Bossink [38]) and examine a wide range of factors that affect the implementation and diffusion of innovations (e.g. Peansupap and Walker [39]). However, the concept of organisational readiness for innovation so far has not been fully investigated by researchers in this area. Given this gap, the following research questions were formulated:

- RQ1: What is the overall level of Innovation Diffusion Readiness (IDR) among AED firms?

- RQ2: Do distinct IDR clusters exist among AED firms?

- RQ3: If such clusters exist, do AED firms belonging to different IDR clusters perform differently in terms of innovation diffusion?

The following section describes the research method employed to help answer the above research questions.

\section{Research methodology}

\subsection{Measures}

As suggested by Patterson et al. [40], the measurement of climate is generally conducted primarily via quantitative-based questionnaire applied comparatively across several organisations. In this study, IDR was conceptualised based on the concept of climate for innovation. Therefore, the questionnaire survey research approach was deemed appropriate for assessing a firms' level of IDR. To achieve this, the present research adapted the refined survey instrument for evaluating climate for innovation and its outcomes in Australian AED firms developed by Panuwatwanich [30]. This survey instrument was 
validated through a confirmatory factor analysis (CFA) and consisted of five constructs: (1) leadership for innovation; (2) team climate for innovation; (3) organisational culture for innovation; (4) innovation diffusion outcomes; and (5) business performance. Table 1 presents the details of the factors that represent each of these constructs. For the purpose of this research, only the scales for the first four constructs were adopted. The measures of leadership, team climate and organisational culture for innovation were combined to represent the IDR scale, whereas the measure of innovation diffusion outcomes (IDO) was used to serve as a performance criterion to help determine if there was any association between the levels of IDR and the ability to diffuse innovations among the sampled firms. The types of innovations included in this instrument are product (advanced technologies and innovative design solutions) and process (innovative design practices). In total the questionnaire consisted of 49 items, all measured using a five-point Likert scale. These items made up 13 factors, with 10 factors representing the IDR scale and three factors signifying the resulting performancebased IDO scale. Descriptions of these factors are presented in Table 2.

<Insert Table 1>

<Insert Table 2>

\subsection{Sample}

A random sample of 110 Australian AED firms was compiled from approximately 300 firms whose main services were in the areas of building and infrastructure, as listed in the Association of Consulting Engineers Australia's directory and the Architectural Information Services database. A survey was carried out in the first quarter of 2009. A package containing five sets of questionnaire, a cover letter and a reply paid envelope was mailed to each of the sampled firms. Of the 110 surveys sent, 54 firms responded. However, a minimum of two individual respondents was required for each firm to be included in the analysis, thus reducing the number of usable responses to 45 , equating to an effective response rate of $41 \%$. The number of individual responses in each firm ranged from 2 to 5 , with an average of 3.6. 


\subsection{Analysis approach}

This research followed a multi-level analysis approach [41], consisting of individual respondent and firm level analyses. Individual respondent analysis aimed to examine the basic statistical properties of the sample and the reliability of the survey instrument (as data collection was conducted with individual design professionals), whereas the firm level analysis provided a means to answer the above listed research questions (which were focused at the firm level) primarily through the use of the cluster analysis technique. The following section provides further details of data analyses and associated results.

\section{Analysis and results}

4.1 Preliminary analysis and data preparation

\subsubsection{Descriptive statistics}

In total, 160 individual survey responses representing 45 AED firms were included in the analysis. The majority of the respondents were civil and structural engineers (40.2\%) and building architects (38.3\%) with approximately $70 \%$ being at the experienced level, having more than six years of experience in their field. The majority of the respondents (79.0\%) also had at least Bachelor's degree.

Preliminary analysis was conducted by screening the dataset of the above individual responses to detect outliers and assess normality through the inspection of skewness and kurtosis. Based on the analysis, no excessive outliers were present and all variables (survey items) were found to have both skewness and kurtosis values within \pm 2.00 , suggesting an acceptable level of normality [42]. The reliability of the measurement scale used in the questionnaire was then examined. Cronbach's Alpha coefficient of reliability of 0.93 calculated from the complete set of items (49 items) indicated a high level of internal consistency for the scale used in the survey [43]. In addition, the internal consistency of both IDR (35 items) and IDO sub-scales (14 items) was also high, having the reliability coefficients (computed separately for each sub-scale) of 0.93 and 0.77 , respectively.

\subsubsection{Firm level data aggregation}

To answer the research questions, analysis at the firm level was required in this study. The individual responses obtained from the questionnaire survey therefore needed to be aggregated to 
represent a single rating for their respective firm for each of the survey items by using mean values. To justify the aggregation, inter-rater agreement was assessed to determine the extent to which individual respondents within each firm agreed on the rating of each item in the survey. The aim of this analysis procedure was to ensure that these aggregated (mean) scores could be appropriately used to represent various measured aspects of each firm. This was carried out using $r_{w g(J)}$, an index of within-group inter-rater agreement for multi-item ratings developed by James et al. [44]. This index was calculated using Equation (1) derived by LeBreton et al. [45]:

$$
r_{w g(J)}=\frac{J\left(1-\frac{\bar{s}_{X}^{2}}{s_{E U}^{2}}\right)}{J\left(1-\frac{\bar{s}_{X}^{2}}{s_{E U}^{2}}\right)+\frac{\bar{s}_{X}^{2}}{s_{E U}^{2}}}
$$

Where:

$J$ is the number of items in the scale;

$\bar{s}_{x}^{2}$ is the obtained average variance of the items in the scale; and

$s_{E U}^{2}$ is the variance of the uniform distribution (=2.0 for the typical five-point Likert scale).

To determine whether the individual responses within each firm could be appropriately aggregated, the $r_{w g(J)}$ cut-off of 0.70 recommended by Dixon and Cunningham [41] was adopted. Using the above equation, the $r_{w g(J)}$ values were calculated separately for both IDR and IDO scales. For the IDR scale, the final $r_{w g(J)}$ values calculated for all 45 firms ranged from 0.94 to 1.00 with an average of 0.98. Being greater than 0.70 , these values suggested that individual respondents collectively agreed on the IDR aspect of their firm across all items. For the IDO scale, all of the $r_{w g(J)}$ values were greater than the cut-off value of 0.70 , except for one firm having a value of $0.11\left(r_{w g(J)}\right.$ range: $\left.0.11-1.00\right)$. This particular firm was therefore removed from the sample group as the results indicated that there was a lack of consensus on the rating of the IDO aspect among the individual respondents from this firm. As a result, a total of 44 sampled firms were included in the final dataset for further analysis.

The final sample of 44 firms (consisting of 158 individual respondents) included in the research equally consisted of firms providing engineering (47.7\%) and architectural (52.3\%) design services, with more than half being small-to-medium size firms (10-200 employees, 61.4\%). The majority of these firms were multinational (40.9\%), followed by national (27.3\%), with the remaining 
being regional (11.4\%) and local (20.5\%). Additionally, more than half of them reported that they have been operating for more than 20 years (59.1\%).

It should be noted that, in addition to individual responses for each item being aggregated to produce firm-level responses, mean values of the aggregated items were calculated to represent a single score of their associated factor. This type of aggregation was justified based on the construct validity of the survey instrument established in the previous research [30].

Table 3 presents the descriptive statistics of the firm-level data, which includes the mean and standard deviation for each of the 13 factors as well as the mean and range of the $r_{w g(J)}$ values based on the final sample $(\mathrm{n}=44)$. It can be seen from the table that the mean values and ranges of the $r_{w g(J)}$ for both the IDR and IDO scales of the final sample are very high, justifying the use of firm-level dataset in this analysis. The mean values of the IDR factors presented in the table ranges from 3.10 to 4.15; this provides an answer to the first research question by suggesting that the overall level of IDR among the sampled Australian AED firms are moderate to high. In addition, it was found that the mean values of two of the IDO factors, namely, IDO2 (innovative design practices) and IDO3 (advanced technology utilisation), are however slightly lower than the moderate level of 3.00.

<Insert Table 3>

4.2 Cluster analysis

To answer the second research question, cluster analysis was adopted to determine whether distinct clusters exist among the sampled firms based on common IDR levels. According to Hair et al. [43], cluster analysis is an exploratory data analysis tool for solving classification problems, aiming to categorise cases into groups or clusters so that each case is very similar to others in its cluster. Two major stages of cluster analysis application were carried out in this research: (1) partitioning; and (2) interpretation. The partitioning stage is the process of determining the number of clusters that may be developed. The interpretation stage is the process of understanding the characteristics of each cluster and developing a name or label that appropriately defines its nature [43]. SPSS version 19 for Windows was employed to perform the analysis. The following sections present further details of the analysis and associated results. 


\title{
4.2.1 Number of clusters
}

There are two main procedures commonly used for cluster analysis - hierarchical and non-hierarchal. The former involves a combination of the objects into clusters through construction of a tree-like structure (dendrogram) depicting the formation of the clusters, whereas the latter (also referred to as K-means) procedure utilises a set of cluster seeds (pre-specified number of clusters) to group objects within a pre-specified distance of the seeds [43,46]. Following Hair et al. [43] and Yeung et al. [47], both procedures were carried out sequentially to identify and produce the major clusters that existed among the sampled firms based on their IDR levels. Hierarchical procedure was employed to examine the number of clusters that should be formed and the non-hierarchical method was applied to produce the actual clusters that contain the details of the associated cluster members [47].

As the first stage of the analysis, the hierarchical procedure was conducted to examine the number of clusters based on all the IDR factors. As describe earlier, this clustering procedure involves a combination of the objects into a hierarchy or a treelike structure, as represented by a dendrogram. A dendrogram provides an indication of heterogeneity change by inspecting average within-cluster distance between all possible combinations of clusters. A decision on the final number of clusters is usually based on the combinations that do not yield a substantial increase in heterogeneity [43]. From an inspection of the dendrogram (Fig. 1), it was found that a division of either two or three clusters could represent the appropriate solutions. However, the three-cluster solution seemed to allow for a more detailed classification that would lead to a more meaningful comparison and interpretation [47], hence the preferred solution.

\author{
$<$ Insert Fig. 1 $>$
}

By using the number of clusters generated from the above analysis as an input, a nonhierarchical (K-means) cluster analysis was undertaken as the second analysis stage to produce the final clusters, which include the details of the firms within each cluster, and their associated centroids for each IDR factor. It should be noted that cluster centroids are in fact the mean values for each item that represent the general characteristics of a cluster [47]. The final centroids (mean values) of the three clusters, based on all the IDR factors, are plotted in Fig. 2. In addition, One-Way Analysis of 
Variance (ANOVA) was conducted which showed that the final centroids of all clusters were significantly different across all factors, thus confirming the validity of the cluster analysis results, as presented in Table 4.

$<$ Insert Fig. 2>

$<$ Insert Table 4>

\subsubsection{Interpretation of clusters}

The centroids plot illustrated in Fig. 2 clearly shows that all three firm groups are clustered upon three different levels across the 10 IDR factors. According to Table 4, Cluster $1(n=9)$ consists of centroid values ranging from 3.54 to 4.52 , with the majority being greater 4.00, hence labelled Very High IDR (VIDR). Cluster $2(\mathrm{n}=25)$ was labelled High IDR (HIDR) as indicated by its centroid values ranging from 3.16 to 4.13 , with most values being close to 4.00. Lastly, Cluster $3(\mathrm{n}=10)$ was labelled Moderate IDR (MIDR) based on most of its centroids concentrating around the middle value of 3.00, ranging from 2.74 to 3.80 . For referencing purposes, these cluster labels (i.e. VIDR, HIDR and MIDR) have been applied to represent the three firm groups, from this point onward for further interpretation and discussion.

To better understand the identified clusters, characteristics of the firms within each cluster were examined with the goal to extract any distinctive features that could explain each of the three firm groups. Table 5 presents a summary of demographic information for all the three clusters. The table shows the number and percentage of firms within each cluster with respect to four characteristic categories: firm type, years in operation, location of operation and firm size. These firm characteristics were examined separately for each of the three clusters, with the aim to identify any association between cluster membership and firm characteristics. For each category, the proportions of firms within each characteristic were compared across all clusters, with respect to those of the entire sample (as reported in Section 4.1.2).

$<$ Insert Table 5> 
From inspection of the table, it is evident that the proportions of each demographic category within the HIDR group are fairly consistent with those of the total sample. This suggests that this group could be treated as a baseline reference for comparison. By examining patterns of characteristics proportions for all clusters in Table 5, firm type and firm size appear to have some relationships with the different levels of IDR. For firm type, it can be seen that the VIDR group consists of more engineering firms than architectural firms. For the lower IDR group (MIDR), the proportion of engineering firms is much lower. In terms of firm size, the proportions of firms with more than 200 employees tend to increase with the improvement in the levels of IDR. For the remaining two demographic characteristics examined, their proportion patterns do not seem to indicate any association with the different levels of IDR.

Analysis was further conducted to answer the last research question, which sought to determine if all of the three different clusters differed in terms of their IDO levels. To achieve this, the overall mean value of each IDO factor was first calculated for each of the identified clusters (i.e. the IDO factor's mean values of all the firms within each of the three clusters were averaged). ANOVA was then conducted by using the cluster membership (i.e. cluster 1, 2 or 3 ) as an independent variable and the three IDO factors as dependent variables. Table 6 presents the results of this analysis along with the mean values of all the three IDO factors for each of the three clusters. A graphical comparison of these mean values is also illustrated in Fig. 3. The results presented in Table 6 show that only the mean scores of the IDO2 factor from the three different clusters differs significantly $(\mathrm{F}=$ 14.835, Sig.= 0.000), with the VIDR cluster having the highest mean value while the MIDR having the lowest. This implies that firms within a group with a higher level of IDR tend to have a higher level of innovative design practices. No significant differences in the levels of innovative design solutions (IDO1) and advanced technology utilisation (IDO3) were found among the different IDR clusters.

$$
\begin{aligned}
& <\text { Insert Table 6> } \\
& \text { < Insert Fig. 3> }
\end{aligned}
$$

Because there is an indication of a possible influence of demographical factors (firm type and firm size) on IDO, further analysis using Factorial (Three-Way) ANOVA was conducted to examine the combined effects of IDR clusters, firm type and firm size on different IDO factors. According to the 
Factorial ANOVA results presented in Table 7, it can be seen that there is no statistically significant difference in all IDO factors among firms having different firm type and size. The only significant relationship seems to be of that between the IDR clusters and IDO2 $(F=7.044$, Sig. $=0.006)$, which is consistent with the findings from the One-Way ANOVA presented above. Furthermore, the nonsignificant interaction terms for all the IDO factors suggest that, regardless of the IDR clusters, firms having different sizes and types do not perform significantly differently across all the IDO factors.

$<$ Insert Table 7>

\section{Discussion and conclusion}

The primary aim of this research was to evaluate IDR among Australian AED firms. For the purpose of this research, IDR was conceptualised on the basis of three socio-psychological constructs identified by Panuwatwanich [30], namely Leadership for Innovation, Team Climate for Innovation and Organisational Culture for Innovation. Through the formulation of three research questions, the present research sought to: assess the overall level of IDR for the sampled Australian AED firm; identify whether distinct clusters of firms with a similar IDR level existed; and to determine whether each IDR cluster performed differently in terms of IDO.

To achieve the research aim, a survey was conducted with individual professionals employed in AED firms within Australia. The analytical approach employed in this research is innovative in that the individual responses from each firm were aggregated and inter-rater agreement was assessed to ensure that the aggregated responses reliably represent firm-level data. The aggregated firm-level dataset were then used in the subsequent cluster analysis stage to explore and identify possible firm clusters. One-Way and Factorial ANOVA were also carried out together with the results from cluster analysis to help answer the research questions.

The data collected from 160 AED professionals were aggregated and analysis conducted at the firm level, with a final sample set of 44 firms included. Assessing the mean values of the 10 IDR factors, it was found that the overall IDR level of the sampled firms was moderate to high. This finding provides an answer to the first research question on the overall level of IDR among Australian AED firms. Cluster analysis results provided an answer to the second research question by revealing 
that these AED firms could be grouped into three main clusters based on their respective IDR levels, namely: Very High IDR (VIDR), High IDR (HIDR) and Moderate IDR (MIDR). It was also found that more than half of the firms fell within the HIDR cluster. Further examination of the demographical characteristics also revealed that firm type and size showed some association with the change in the levels of IDR, where the proportions of engineering firms and firms with more than 200 employees tended to increase with the higher IDR clusters. These findings suggest that higher IDR appears to present in firms with larger size, and in engineering firms compared with architectural firms. In fact, firm size and firm type have been identified in past research as one of the determinants of innovation. For example, Arias-Aranda et al. [48] found that the degree of innovation is positively associated with firm size according to their research conducted with engineering consulting firms in Spain. With respect to firm type, a study by Ankrah and Langford [49] on the comparison of organisational culture between contractors and architects seem to shed some light on certain organisational aspects that may relate to the understanding of IDR within the context of this study. Nonetheless, the present study found that the difference in firm type and size has no bearing on firms' IDO performance.

Providing an answer to the last research question, the analysis results indicated that clusters with higher IDR performed significantly better in terms of innovative design practices (i.e. more extensive use of value-based design techniques/concepts). This shows that firms shifting to a higher IDR cluster could achieve a significant improvement in the diffusion of this type of process innovation. However, this was not evidently the case for innovative design solutions and the utilisation of advanced technologies, where there were no significant differences between the three clusters. These findings may be explicated by: the nature of value-based design techniques and concepts (e.g. Quality Function Deployment and Value Management) that tends to require more collaborative effort from team members to successfully implement and incorporate such techniques/concepts into their standard design practices; and the dimensions of IDR are conceptualised upon the socio-psychological constructs. To further elaborate, Dikmen et. al. [50] argue that a successful implementation of Quality Function Deployment largely hinges upon effective cross-functional communications. Similarly, Green [51] asserts that Value Management provides a framework for facilitating thought and communication, underpinned by shared value and understanding to establish shared social reality, 
which is necessary to successfully achieve common objectives. As such, the socio-psychological aspects of the DOI theory become highly relevant in this regard. A firm, as a social system, that has a higher level of IDR will most likely have a social structure and systems norms that are more conducive to effective communication and sharing of information among members of the firm. Therefore, firm groups with higher levels of IDR are more likely to have a better diffusion of innovative design practices. The findings imply that the IDR scale used in this research may be more relevant to the diffusion of "value-based" innovation as opposed to the remaining two IDO factors that are more product/technological oriented. Such value-based innovations are generally more concerned with new strategies, techniques or procedures implemented to enhance value for the clients, stakeholders as well as the project supply chain.

The diffusion of advanced technologies factor within the IDO scale was more focused on the use of computer technologies to facilitate the design process. The diffusion of such technologies (i.e. ICT) has been found in past research to be influenced by specific technology characteristics associated with the ease of use and functionality of those technologies [29]. Moreover, the level of advanced technologies utilisation and diffusion has also been found to be explained by bandwagon pressure [52] and Technology Acceptance Model (TAM), where the actual use of such technologies is mainly influenced by users' perceived usefulness and ease of use [53]. In the case of bandwagon pressure, although the socio-psychological effect may play a role in the diffusion of advanced technologies, the locus of such pressure generally relates to external variables rather than those within a firm. According to Abrahamson and Rosenkopt [54], "Bandwagons are diffusion processes whereby organizations adopt an innovation, not because of their individual assessments of the innovation's efficiency and returns, but because of a bandwagon pressure caused by the sheer number of organizations that have already adopted this innovation” (p. 488). This implies that the diffusion of advanced technologies within the firm may be less likely to be influenced by the firm's IDR alone. External factors may have a joint contribution in this respect. Turning to innovative design solutions, although it has been widely acknowledged that the conception of good design ideas is often a result of deep collaboration among team members under innovation-conducive environments [55], this factor could be dependent very much upon the opportunity for the project team to innovate, which is invariably determined by such factors as types of project and client requirements, rather than the actual IDR levels alone [56]. In 
addition, further analysis showed that there are no significant relationships between firm type and size and firms' IDO performance. Further research may be required to provide a better understanding on the influence of such firm characteristics within this research field.

In summary, this research examined the concept of IDR and indentified three different clusters of Australian AED firms according to three main IDR levels. The findings also show that there is a statistically significance relationship between firms in higher IDR clusters and their stronger performance in terms of the diffusion of innovative design practices. While it is argued that the approach taken to assess the IDR within the context of this study was more appropriate than the reported readiness assessment frameworks reviewed earlier in this paper, the developed IDR scale seems to be more suitable for examining the diffusion of value-based innovations rather than product/technological innovations within an AED firm.

\section{Future research}

Demographic characteristic factors that appeared to be associated with the three levels of IDR were identified, including firm size and type. Clearly, such factors together with other demographical factors could be further investigated in future research and incorporated into the interpretation of cluster solutions. In this way, the characteristics of each firm cluster can be better understood and an extensive cluster profile can be created. Such development of more extensive cluster profiles would enhance the IDR assessments and allow for benchmarking activities to be conducted. Finally, based on such cluster profiles, descriptive maturity levels of IDR should also be developed to provide meaningful information on the existing IDR level of the firm being assessed as well as what has to be done for the firm to progress into a higher IDR maturity level (i.e. firm to move from MIDR to HIDR). It is also worth investigating other potential external variables that may have an impact on a firm's IDR and how this can, in turn, affect the diffusion of different types of innovations.

\section{References}

[1] R. Torbett, A.J. Salter, D.M. Gann, M. Hobday, Design Performance Measurement in the Construction Sector: A Pilot Study, Science and Technology Policy Research, University of Sussex, 2001.

[2] A. Milne, L. Leifer, The ecology of innovation in engineering design, Proceedings of the International Conference on Engineering Design (ICED 99), Munich, 1999.

[3] A. Salter, D. Gann, Sources of ideas for innovation in engineering design, Research Policy 32 (32) (2003) 1309-1324. 
[4] K. Panuwatwanich, R.A. Stewart, S. Mohamed, The role of climate for innovation in enhancing business performance: the case of design firms, Engineering, Construction and Architectural Management 15 (5) (2008) 407-422.

[5] J.M. Kamara, G. Augenbore, C.J. Anumba, P.M. Carrillo, Knowledge management in the architecture, engineering and construction industry, Construction Innovation 2 (1) (2002) 5367.

[6] C.E. Siemieniuch, M.A. Sinclair, A framework for organisational readiness for knowledge management, International Journal of Operations and Production Management 24 (1) (2004) 79-98.

[7] M.M.A. Khalfan, C.J. Anumba, P.M. Carrillo, Readiness assessment for concurrent engineering in construction, in: J.M.K.a.A.-F.C.-D. Chimay J. Anumba (Ed.), Concurrent Engineering in Construction Projects Taylor and Francis, New York, 2007, pp. 30-56.

[8] V. Peansupap, D. Walker, Constraints on the ICT diffusion within large Australian construction firms Proceedings of the International Symposium on Globalisation and Construction 2004, The International Council on Innovation and Research in Building and Construction (CIB), Bangkok, 2004.

[9] K. Ruikar, C.J. Anumba, P.M. Carrillo, VERDICT - An e-readiness assessment application for construction companies, Automation in Construction 15 (1) (2006) 98-110.

[10] E.M. Rogers, Diffusion of Innovations, 5th ed., Free Press, New York, 2003.

[11] A. Zerfass, Innovation readiness: a framework for enhancing corporations and regions by innovation communication, Innovation Journalism 2 (8) (2005) 3-27.

[12] N.R. Anderson, M.A. West, Measuring climate for work group innovation: development and validation of the team climate inventory, Journal of Organizational Behavior 19 (3) (1998) 235-259.

[13] P.G. Bain, L. Mann, A. Pirola-Merlo, The Innovation imperative: the relationships between team climate, innovation, and performance in research and development teams, Small Group Research 32 (1) (2001) 55-73.

[14] C.O. Egbu, J. Henry, G.R. Kaye, P. Quintas, T.R. Schumacher, B.A. Young, Managing organizational innovations in construction, in: W. Hughes (Ed.), Proceedings of the 14th Annual Conference of the Association of Researchers in Construction Management (ARCOM), Reading, 1998, pp. 605-614.

[15] G. Allport, The Nature of Prejudice, Addison-Wesley, Cambridge, 1954.

[16] A. Hartmann, The role of organizational culture in motivating innovative behaviour in construction firms, Construction Innovation 6 (3) (2006) 159-172.

[17] S.W.J. Kozlowski, M.L. Doherty, Integration of climate and leadership: examination of a neglected issue, Journal of Applied Psychology 74 (4) (1989) 546-553.

[18] S.G. Scott, R.A. Bruce, Determinants of innovative behavior: a path model of individual innovation in the workplace, Academy of Management Journal 37 (3) (1994) 580-607.

[19] B.E. Ashfort, Climate formation: issues and extensions, The Academy of Management Review 10 (4) (1985) 837-847.

[20] T.M. Amabile, R. Conti, H. Coon, J. Lazenby, M. Herron, Assessing the work environment for creativity, The Academy of Management Journal 39 (5) (1996) 1154-1184.

[21] M.F. Dulaimi, M.P. Nepal, M. Park, A hierarchical structural model of assessing innovation and project performance, Construction Management and Economics 23 (6) (2005) 565-577.

[22] S. Martin, D. Beimborn, M.A. Parikh, T. Weitzel, Organizational readiness for business process outsourcing: a model of determinants and impact on outsourcing success, Proceedings of the 41st Hawaii International Conference on System Sciences, IEEE, Hawaii, 2008, pp. 374-383.

[23] G. Zaltman, R. Duncan, J. Holbek, Innovations and Organizations, Wiley, New York, 1973.

[24] C. Cherniss, Emotional intelligence and organizational effectiveness, in: C. Cherniss, D. Goleman (Eds.), The Emotionally Intelligent Workplace: How to Select for, Meaure and Improve Emotional Intelligece in Individuals, Groups, and Organizations, Jossey-Bass, San Francisco, 2001.

[25] R. Snyder-Halpern, Indicators of organizational readiness for clinical information technology/systems innovation: a Delphi study, International Journal of Medical Informatics 63 (3) (2001) 179-204. 
[26] T. Greenhalgh, K. Stramer, T. Bratan, E. Byrne, Y. Mohammad, J. Russell, Introduction of shared electronic records: multi-site case study using diffusion of innovation theory, British Medical Journal doi: 10.1136/bmj.a1786 (2008).

[27] S. Hamilton, S. McLaren, A. Mulhall, Assessing organisational readiness for change: use of diagnostic analysis prior to the implementation of multidisciplinary assessment for acute stroke care, Implementation Science 2 (21) (2007) doi:10.1186/1748-5908-1182-1121.

[28] W.E.K. Lehman, J.M. Greener, D. Simpson, Assessing organizational readiness for change, Journal of Substance Abuse Treatment 22 (4) (2002) 197-209.

[29] V. Peansupap, D. Walker, Exploratory factors influencing information and communication technology diffusion and adoption within Australian construction organizations: a micro analysis, Construction Innovation 5 (3) (2005) 135-157.

[30] K. Panuwatwanich, Modelling the Innovation Diffusion Process in Australian Architectural and Engineering Design Organisations, PhD Thesis, Griffith School of Engineering, Griffith School of Engineering, Griffith University, 2008.

[31] J.R. Cooper, A multidimensional approach to the adoption of innovation, Management Decision 36 (8) (1998) 493-502.

[32] R.W. Zmud, Diffusion of modern software practices: influence of centralization and formalization, Management Science 28 (12) (1982) 1421-1431.

[33] F.Y.Y. Ling, Managing the implementation of construction innovations, Construction Management and Economics 21 (6) (2003) 635-649.

[34] G. Seaden, M. Guolla, J. Doutriaux, J. Nash, Strategic decisions and innovation in construction firms, Construction Management and Economics 21 (6) (2003) 603-612.

[35] M. Sexton, P. Barrett, Performance-based building and innovation: balancing client and industry needs, Building Research \& Information 33 (2) (2005) 142-148.

[36] L. Koskela, R. Vrijhoef, Is the current theory of construction a hindrance to innovation?, Building Research \& Information 29 (3) (2001) 197-207.

[37] G. Winch, Zephyrs of creative destruction: understanding the management of innovation in construction, Building Research \& Information 26 (4) (1998) 268-279.

[38] B.A.G. Bossink, Managing drivers of innoyation in construction networks, Journal of Construction Engineering and Management 130 (3) (2004) 337-345.

[39] V. Peansupap, D.H.T. Walker, Factors enabling information and communication technology diffusion and actual implementation in construction organisations, Electronic Journal of Information Technology in Construction 10 (2005) 193-218.

[40] M.G. Patterson, M.A. West, V.J. Shackelton, J.F. Dawson, R. Lawthom, S. Maitlis, D.L. Robinson, A.M. Wallace, Validating the organizational climate measure: links to managerial practices, productivity and innovation, Journal of Organizational Behavior 26 (4) (2005) 379408.

[41] M.A. Dixon, G.B. Cunningham, Data aggregation in multilevel analysis: a Review of conceptual and statistical issues, Measurement in Physical Education and Exercise Science 10 (2) (2006) 85-107.

[42] S.G. West, J.F. Finch, P.J. Curran, Structural equation models with nonnormal variables: problems and remedies, in: R.H. Hoyle (Ed.), Structural Equation Modeling: Concepts, Issues, and Applications, SAGE Publications, Thousand Oaks, 1995.

[43] J.F. Hair, W.C. Black, B.J. Babin, R.E. Anderson, R.L. Tatham, Multivariate Data Analysis, 6th edn, Pearson Prentice Hall, Upper Saddle River, N.J, 2006.

[44] L.R. James, R.G. Demaree, G. Wolf, Estimating within-group interrater reliability with and without response bias, Journal of Applied Psychology 69 (1) (1984) 85-98.

[45] J.M. LeBreton, L.R. James, M.K. Lindell, Recent Issues Regarding rWG, rWG, rWG(J), and rWG(J), Organizational Research Methods 8 (1) (2005) 128-138.

[46] D.J. Ketchen, C.L. Shook, The application of cluster analysis In strategic management research: an analysis and critique, Strategic Management Journal 17 (6) (1996) 441-458.

[47] A.C.L. Yeung, L.Y. Chan, T.S. Lee, An empirical taxonomy for quality management systems: a study of the Hong Kong electronics industry, Journal of Operations Management 21 (1) (2003) 45-62.

[48] D. Arias-Aranda, B. Minguela-Rata, A. Rodriguez-Duarte, Innovation and firm size: an empirical study for Spanish enginering consulting companies, European Journal of Innovation Management 4 (3) (2001) 133-141. 
[49] N.A. Ankrah, D.A. Langford, Architects and contractors: a comparative study of organizational cultures, Construction Management and Economics 23 (2005) 597-607.

[50] I. Dikmen, M.T. Birgonul, S. Kiziltas, Strategic use of quality function deployment (QFD) in the construction industry, Building and Environment 40 (2) (2005) 247-255.

[51] S.D. Green, Beyond value engineering: SMART value management for building projects, International Journal of Project Management 12 (1) (1994) 49-56.

[52] S. Kale, D. Arditi, Diffusion of computer aided design technology in architectural design practice, Journal of Construction Engineering and Management 131 (10) (2005) 1135-1141.

[53] F.D. Davis, R.P. Bagozzi, P.R. Warshaw, User acceptance of computer technology: a comparison of two theoretical models, Management Science 35 (8) (1989) 982-1003.

[54] E. Abrahamson, L. Rosenkopf, Institutional and competitive bandwagons: using mathematical modeling as a tool to explore innovation diffusion, Academy of Management Review 18 (3) (1993) 487-517.

[55] A. Salter, R. Torbett, Innovation and performance in engineering design, Construction Management and Economics 21 (6) (2003) 573-580.

[56] K. Panuwatwanich, R.A. Stewart, S. Mohamed, Critical pathways to enhanced innovation diffusion and business performance in Australian design firms, Automation in Construction 18 (6) (2009) 790-797. 


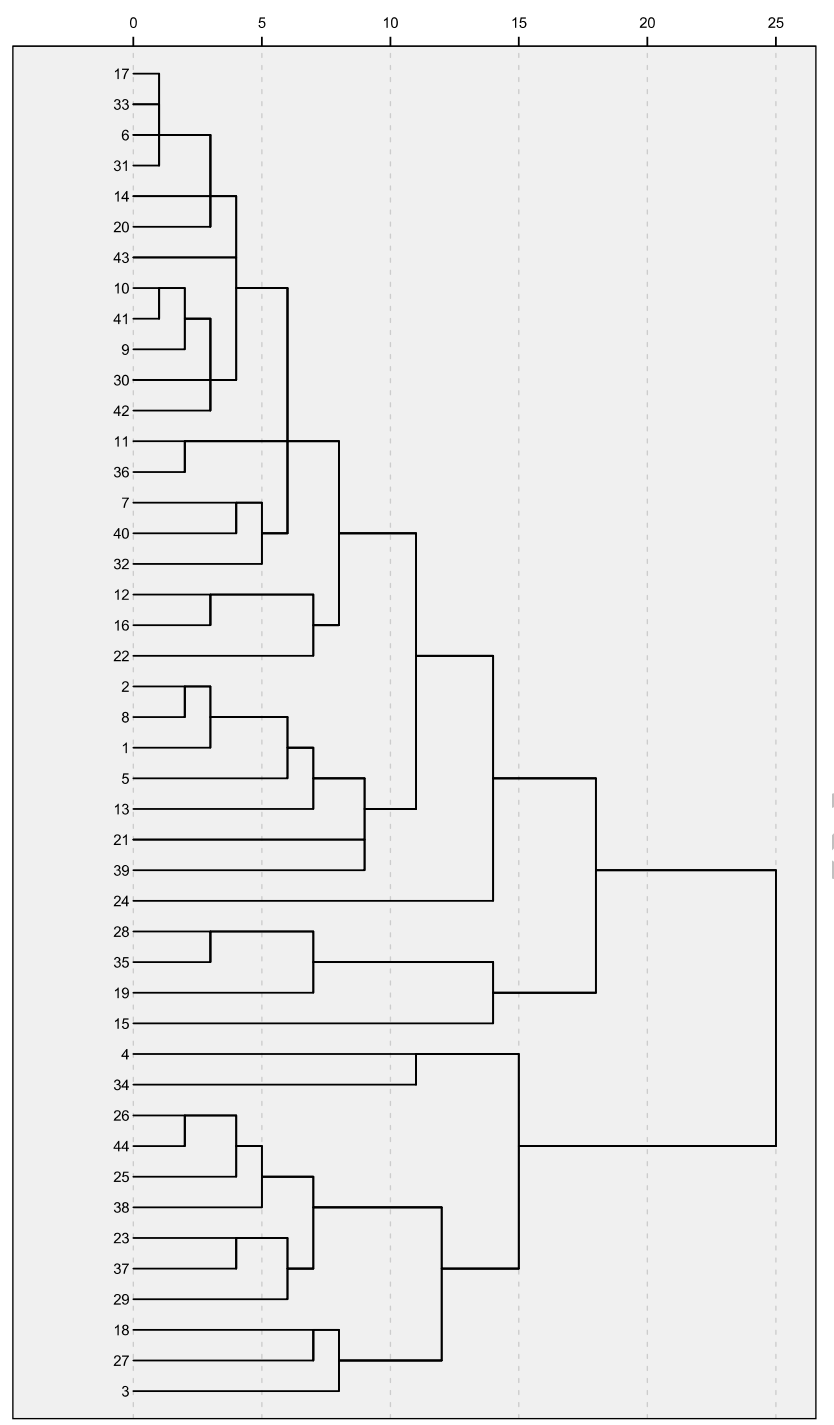

Fig. 1. Dendrogram

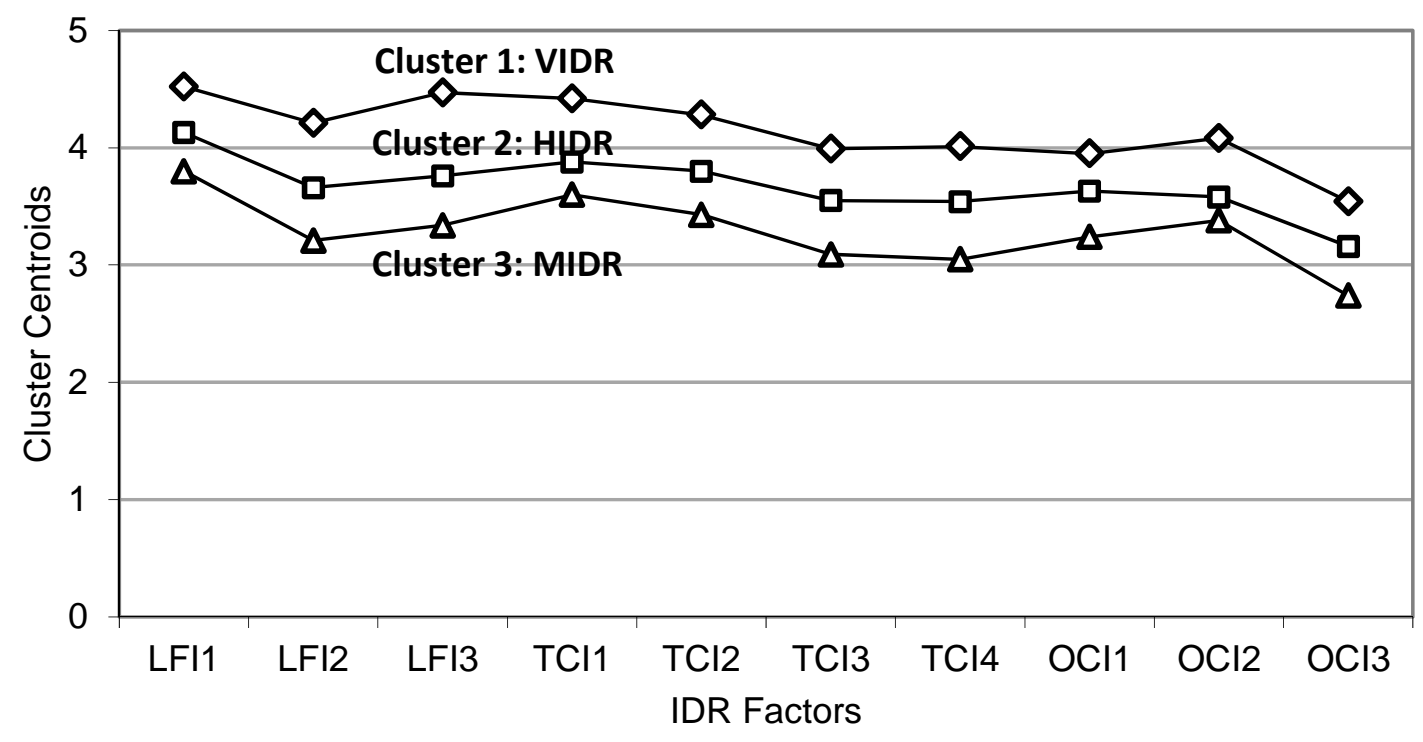

Fig. 2. Centroid plots of the three clusters 


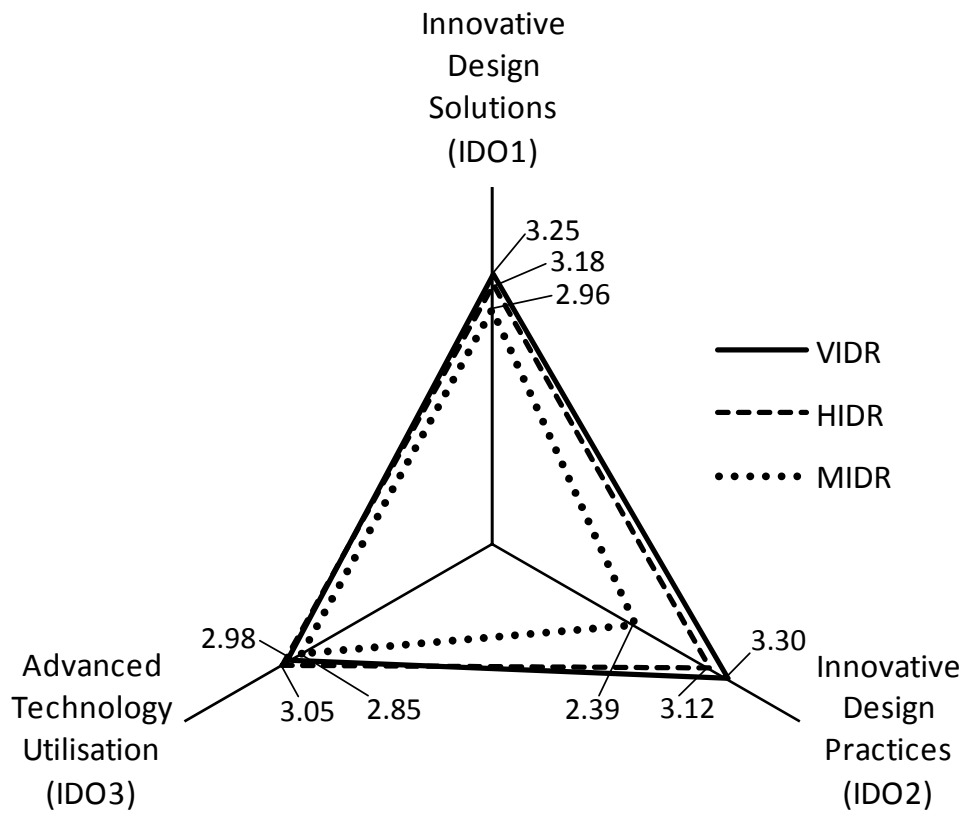

Fig. 3. IDO factors comparison between the three clusters 
Table 1. Model constructs and factor details [4]

\begin{tabular}{ll}
\hline Construct & Factor details \\
\hline 1. Leadership for innovation (LFI) & LFI1: Innovation championing \\
& LFI2: Creativity stimulation and inspiration \\
& LFI3: Engagement and support \\
& TCI1: Vision \\
2. Team climate for innovation (TCI) & TCI2: Participative safety \\
& TCI3: Task orientation \\
& TCI4: Support for innovation \\
3. Organisational culture for innovation (OCI) & OCI1: Propensity for creativity \\
& OCI2: Freedom and autonomy \\
& OCI3: Innovation support and facilitation \\
4. Innovation diffusion outcomes (IDO) & IDO1: Innovative design products \\
IDO2: Innovative design practices \\
IDO3: Advanced technology utilisation \\
B. Business performance (BPM) & BPM2: Client satisfaction \\
\hline
\end{tabular}


Table 2. Factor descriptions (adapted from Panuwatwanich [30])

\begin{tabular}{l}
\hline Factor \\
\hline Innovation Diffusion Readiness (IDR) \\
LFI1: Innovation championing (3 items) \\
LFI2: Creativity stimulation and inspiration (4 \\
items)
\end{tabular}

LFI3: Engagement and support (5 items)

TCI1: Vision (4 items)

TCI2: Participative safety (3 items)

TCI3: Task orientation (2 items)

TCI4: Support for innovation (3 items)

OCI1: Propensity for creativity (5 items)

OCI2: Freedom and autonomy (2 items)

OCI3: Innovation support and facilitation (4 items)

Innovation Diffusion Outcomes (IDO)

IDO1: Innovative design solutions (5 items)

IDO2: Innovative design practices (4 items)

IDO3: Advanced technology utilisation (5 items)

\section{Description}

The degree to which leaders seek out and promote new ideas, technologies and/or innovative approaches for solving problems.

The degree to which leaders inspire and encourage team members to develop new ideas and/or approaches for solving problems.

The quality of supportive relationships between leaders and their subordinates.

The establishment of a team's clearly defined and shared goal that provides focus and direction to team members as a motivating force at work.

The degree to which involvement in decision making is motivated and reinforced without fear of criticism among team members.

The degree of concern with the quality of task performance in relation to shared vision or outcomes among team members.

The degree of expectation, approval and practical support of any attempts made by team members to introduce new and improved ways of doing things.

The aspect of the firm's culture that is conducive to creativity, in terms of the perceived degree of flexibility, risk orientation, and open collaboration.

The extent to which the firm allows its members to have freedom in carrying out their own work.

The perceived availability of resource in terms of training, manpower, time and money set aside for creative/innovative activities.

The level of innovativeness of the firm's design solutions (e.g. awards, industry recognition, etc.).

The extent to which innovative (value-based) design practices are utilised to facilitate design processes (e.g. value management, constructability review, life cycle costing, etc.).

The level of design technology utilised to facilitate design activities (e.g. design representation, utilisation of advanced software tools, etc.). 
Table 3. Factor means and standard deviations (firm-level; $\mathrm{n}=44$ )

\begin{tabular}{lcccc} 
& \multirow{2}{*}{ Factor } & Mean & S.D. & \multicolumn{2}{c}{$\boldsymbol{r}_{\text {wg(i) }}$} \\
\cline { 4 - 6 } & & & Mean & Range \\
\hline Innovation Diffusion Readiness (IDR) & & & 0.98 & $0.94-1.00$ \\
LFI1: Innovation championing & 4.14 & 0.38 & & \\
LFI2: Creativity stimulation and inspiration & 3.67 & 0.43 & & \\
LFI3: Engagement and support & 3.81 & 0.47 & & \\
TCI1: Vision & 3.93 & 0.38 & & \\
TCI2: Participative safety & 3.81 & 0.43 & & \\
TCI3: Task orientation & 3.54 & 0.38 & & \\
TCI4: Support for innovation & 3.52 & 0.44 & & \\
OCI1: Propensity for creativity & 3.61 & 0.37 & & \\
OCI2: Freedom and autonomy & 3.64 & 0.46 & & \\
OCI3: Innovation support and facilitation & 3.14 & 0.43 & & \\
Innovation Diffusion Outcomes (IDO) & & & 0.90 & $0.80-1.00$ \\
IDO1: Innovative design solutions & 3.15 & 0.40 & & \\
IDO2: Innovative design practices & 2.99 & 0.52 & & \\
IDO3: Advanced technology utilisation & 2.99 & 0.44 & & \\
\hline
\end{tabular}

Table 4. ANOVA results for IDR Factors

\begin{tabular}{|c|c|c|c|c|c|}
\hline \multirow[b]{2}{*}{ Factor } & \multicolumn{3}{|c|}{ Centroids } & \multicolumn{2}{|c|}{ ANOVA test statistics } \\
\hline & $\begin{array}{c}\text { Cluster 1 } \\
\text { VIDR } \\
(\mathbf{n}=9)\end{array}$ & $\begin{array}{c}\text { Cluster } 2 \\
\text { HIDR } \\
(n=25)\end{array}$ & $\begin{array}{c}\text { Cluster } 3 \\
\text { MIDR } \\
(\mathbf{n}=10)\end{array}$ & $\mathbf{F}$ & Sig. \\
\hline LFI1: Innovation championing & 4.52 & 4.13 & 3.80 & 13.808 & 0.000 \\
\hline LFI2: Creativity stimulation and inspiration & 4.21 & 3.66 & 3.21 & 30.405 & 0.000 \\
\hline LFI3: Engagement and support & 4.47 & 3.76 & 3.34 & 39.594 & 0.000 \\
\hline TCI1: Vision & 4.42 & 3.88 & 3.60 & 24.439 & 0.000 \\
\hline TCI2: Participative safety & 4.28 & 3.80 & 3.43 & 15.967 & 0.000 \\
\hline TCI3: Task orientation & 3.99 & 3.55 & 3.09 & 31.515 & 0.000 \\
\hline TCI4: Support for innovation & 4.01 & 3.54 & 3.05 & 23.097 & 0.000 \\
\hline OCI1: Propensity for creativity & 3.95 & 3.63 & 3.24 & 14.618 & 0.000 \\
\hline OCI2: Freedom and autonomy & 4.08 & 3.58 & 3.38 & 7.975 & 0.001 \\
\hline OCI3: Innovation support and facilitation & 3.54 & 3.16 & 2.74 & 12.364 & 0.000 \\
\hline
\end{tabular}


Table 5. Demographic information for IDR clusters

\begin{tabular}{|c|c|c|c|c|c|c|c|c|c|}
\hline \multirow{3}{*}{ Category } & \multirow{3}{*}{ Description } & \multicolumn{6}{|c|}{ Clusters } & & \\
\hline & & \multicolumn{2}{|c|}{ VIDR } & \multicolumn{2}{|c|}{ HIDR } & \multicolumn{2}{|c|}{ MIDR } & \multicolumn{2}{|c|}{ Total } \\
\hline & & $\mathbf{n}$ & $\%$ & $\mathbf{n}$ & $\%$ & $\mathbf{n}$ & $\%$ & $\mathbf{n}$ & $\%$ \\
\hline \multirow[t]{2}{*}{ Firm type } & Architecture & 2 & 22.2 & 13 & 52.0 & 8 & 80.0 & 23 & 52.3 \\
\hline & Engineering & 7 & 77.8 & 12 & 48.0 & 2 & 20.0 & 21 & 47.7 \\
\hline \multirow{4}{*}{$\begin{array}{l}\text { Years in } \\
\text { operation }\end{array}$} & Less than 5 years & 1 & 11.1 & 1 & 4.0 & 1 & 10.0 & 3 & 6.8 \\
\hline & $6-10$ years & 1 & 11.1 & 3 & 12.0 & 0 & 0.0 & 4 & 9.1 \\
\hline & $11-20$ years & 2 & 22.2 & 6 & 24.0 & 3 & 30.0 & 11 & 25.0 \\
\hline & More than 20 years & 5 & 55.6 & 15 & 60.0 & 6 & 60.0 & 26 & 59.1 \\
\hline \multirow{4}{*}{$\begin{array}{l}\text { Location of } \\
\text { operation }\end{array}$} & Local & 1 & 11.1 & 7 & 28.0 & 1 & 10.0 & 9 & 20.5 \\
\hline & Regional & 1 & 11.1 & 4 & 16.0 & 0 & 0.0 & 5 & 11.4 \\
\hline & National & 1 & 11.1 & 6 & 24.0 & 5 & 50.0 & & 27.3 \\
\hline & Multinational & 6 & 66.7 & 8 & 32.0 & 4 & 40.0 & & 40.9 \\
\hline \multirow[t]{2}{*}{ Firm size } & 10-200 employees & 4 & 44.4 & 16 & 64.0 & 7 & 70.0 & & 61.4 \\
\hline & More than 200 employees & 5 & 55.6 & 9 & 36.0 & 3 & 30.0 & 17 & 38.6 \\
\hline
\end{tabular}

Table 6. One-Way ANOVA results (IDR clusters as independent variable)

\begin{tabular}{lcccccc}
\hline \multirow{2}{*}{\multicolumn{1}{c}{ Factor }} & \multicolumn{2}{c}{ IDR clusters mean values } & & \multicolumn{2}{c}{ ANOVA test statistics } \\
\cline { 2 - 3 } & VIDR & HIDR & MIDR & & F & Sig. \\
\hline IDO1: Innovative Design Solutions & 3.25 & 3.18 & 2.96 & & 1.581 & 0.218 (n.s.) \\
IDO2: Innovative Design Practices & 3.30 & 3.12 & 2.39 & & 14.835 & 0.000 \\
IDO3: Advanced Technology Utilisation & 2.98 & 3.05 & 2.85 & & 0.720 & 0.493 (n.s.) \\
\hline
\end{tabular}

Note: n.s. = not significant

Table 7. Factorial ANOVA results

\begin{tabular}{llcc}
\hline \multirow{2}{*}{ Dependent Variable } & \multicolumn{2}{c}{ Independent Variables } & \multicolumn{2}{c}{ ANOVA test statistics } \\
\cline { 2 - 4 } & & F & Sig. \\
\hline IDO1: Innovative Design Solutions & IDR clusters & 1.288 & 0.301 (n.s.) \\
& Firm size & 1.861 & 0.140 (n.s.) \\
& Firm type & 0.671 & 0.424 (n.s.) \\
& Interaction term: IDR clusters*Firm size & 1.051 & 0.443 (n.s.) \\
& Interaction term: IDR clusters*Firm type & 0.022 & 0.885 (n.s.) \\
& Interaction term: Firm size*Firm type & 2.210 & 0.111 (n.s.) \\
& & & \\
& IDR clusters & 7.044 & 0.006 \\
& Firm size & 1.373 & 0.279 (n.s.) \\
& Firm type & 0.393 & 0.539 (n.s.) \\
& Interaction term: IDR clusters*Firm size & 1.656 & 0.177 (n.s.) \\
& Interaction term: IDR clusters*Firm type & 0.486 & 0.495 (n.s.) \\
& Interaction term: Firm size*Firm type & 1.583 & 0.224 (n.s.) \\
& & & \\
& IDR clusters & 1.102 & 0.355 (n.s.) \\
& Firm size & 1.517 & 0.227 (n.s.) \\
& Firm type & 2.949 & 0.104 (n.s.) \\
& Interaction term: IDR clusters*Firm size & 0.291 & 0.968 (n.s.) \\
& Interaction term: IDR clusters*Firm type & 0.000 & 0.989 (n.s.) \\
& Interaction term: Firm size*Firm type & 0.581 & $0.681($ n.s.) \\
\hline
\end{tabular}

Note: n.s. = not significant 


\section{Figure Captions}

Fig. 1. Dendrogram

Fig. 2. Centroid plots of the three clusters

Fig. 3. IDO factors comparison between the three clusters 